\title{
Performatividad estética y política del mundo objetal
}

\section{Aesthetic and politic performativity of the objectal world}

\author{
Adryan Pineda Repizzo \\ Facultad de Ciencias Humanas, Universidad Autónoma de Colombia. Bogotá, \\ Colombia. \\ adryan.pineda@fuac.edu.co
}

\section{Resumen}

Este artículo presenta los resultados de un análisis fundamentado de un caso de organización objetal. Se destaca la relevancia de esclarecer las dinámicas de las relaciones de significación presentes en los modos de apropiación de los objetos al interior del espacio de la sala del hogar, dado que, como muestra el análisis, una determinada disposición de los objetos no puede ser aislada de los procesos de identificación de los individuos y la construcción y comunicación de un modo de ser o un estilo de vida. Para ello, se expone un análisis de caso de organización objetal en relación con teóricos de la cultura contemporánea que han resaltado la importancia de lo estético en la producción y reproducción de prácticas y representaciones culturales. El objetivo es plantear un marco conceptual que aporte al análisis de la estética del mundo objetal en la cultura contemporánea.

Palabras clave: objetos, procesos de identificación, performatividad, estética, cultura material.

\begin{abstract}
This paper presents the results of a grounded analysis of an object organization case. The importance of clarifying the dynamics of the relationships of meaning contained in the modes of appropriation of the objects within the space of the living room is highlighted, since, as the analysis shows, a particular arrangement of objects cannot be isolated from the processes of identification of the individuals and from the construction and communication of a way of being or lifestyle. For this purpose, the case study of object organization is presented in relation to contemporary culture theorists that have highlighted the importance of aesthetics in the production and reproduction of cultural practices and representations. The aim is to propose an alternative framework that enriches the analysis of the aesthetics of the object world in contemporary culture.
\end{abstract}

Keywords: Objects, Identification Processes, Performativity, Aesthetics, Material Culture. 


\section{Performatividad estética y política del mundo objetal}

Las relaciones entre lo estético y lo político suelen ser pensadas en términos macrofísicos. Agenciamientos y regímenes en las artes, retóricas e ideologías en el mercado, entre otros tópicos, han abierto sendos problemas de gran interés para los estudios estéticos contemporáneos. Sin embargo, el problema de la mirada macro es que pasa por alto los efectos pululantes de la gran diversidad de fenómenos que se encuentran en el plano de lo micro y, más aun, de lo más cotidiano. El presente texto es resultado de un esfuerzo por considerar la relación entre lo estético y lo político en el espacio de lo micro y lo cotidiano que, precisamente por su carácter ordinario e inevitable, ha sido desestimado, a saber, el de la cultura material, y dentro de todo lo que abarca este término, aquel conjunto de objetos de uso y modos de organización correspondiente al espacio del hogar que denominamos "sala" o living room. En una de las secciones del Libro de los Pasajes, Walter Benjamin presenta la siguiente nota: "la forma prototípica de todo habitar no es estar en una casa, sino en una funda. Esta exhibe las huellas de su inquilino. En último extremo, la vivienda se convierte en funda" (239). El lugar donde habitamos, y en particular para nuestro caso la sala de nuestro hogar, es un espacio material y simbólico que pone en interacción un conjunto de huellas conformadas por componentes subjetivos, estéticos, sociales y políticos que componen la urdimbre de un rincón íntimo y a la vez socializable, un espacio de reencuentro del sujeto consigo mismo y de conexión con los valores, saberes y significados culturales que construyen y afirman un modo de vida.

En este texto se defenderá que los modos de organización del mundo objetal ${ }^{1}$ responden, reproducen y construyen valores, saberes y significaciones culturales al interior de la vida cotidiana, y que la manera de realización de este fenómeno puede ser descrita en términos de la performatividad de la relación entre el mundo objetal y los procesos de subjetivación marcados por lo estético y lo político. El concepto de performatividad será presentado de manera más atenta más adelante. Por el momento, cabe señalar que este remite a los modos de permanente interacción entre la subjetividad y el mundo material, interacción que se caracteriza por ser mutuamente constitutiva; los procesos de subjetivación se alimentan, estructuran y delimitan en los modos de interacción y organización del mundo objetal. De ahí que, aun cuando se habría podido acudir a otro tipo de organización objetal, la sala es, a nuestro entender, un espacio privilegiado para comprender este fenómeno, en virtud de unas características centrales, a saber: la sala es una especie de portal entre lo público que arriba para entrometerse en lo privado y, a la vez, un límite, una zona de control del intruso; además, el formato de la sala tiende a contar con cierta homogeneidad cultural;

1 Se usará el término "objetal” para hacer referencia a los objetos materiales de uso, a fin de evitar las modulaciones de términos como "objetual", que fácilmente se asocian con el concepto de objetividad o con la referencia a cualquier cosa fuera de o distinta al sujeto. 
y, empero, cada sala se expone al público como un representante de la singularidad de quien habita ese hogar. No cualquier persona puede entrar en la alcoba de un hogar sin pasar por entrometido, mientras que la sala es, por definición, el lugar en el que el extraño es acogido.

Para dar lugar al análisis y al planteamiento del concepto de performatividad objetal, primero examinaremos brevemente un caso particular de organización de los objetos en una sala. En seguida, exploraremos el papel de lo performativo en este modo de organización y finalizaremos con el lugar de las relaciones entre lo estético y lo político en el mundo objetal.

\section{La sala de Lucy²}

Una diversidad de detalles es lo primero que captura la atención en la sala de Lucy. Entrando por una de sus esquinas, aparece un sol de forja, bodegones en las paredes y un impresionante comedor de vidrio templado con sillas de madera, sobre el cual reposa un frutero con uvas artificiales. Ubicado en el mismo punto de visión, una lámpara de pie con forma de palmera en bronce y un bar veneciano de madera incrustada dan paso al juego de sala, compuesto por dos sofás grandes y una mesa de centro grande y maciza, sobre la cual se revela un cenicero en porcelana que también hace las veces de caja musical y un arreglo de flores. A su respaldo, otro cuadro y plantas artificiales ornamentan el lugar. El fondo se muestra, por uno de sus extremos, con un biombo de tiras de bronce trenzadas, un baúl canadiense, una pequeña mesa hindú, un bar de Venecia y una estatuilla en bronce. Tanto este bar como el anterior están llenos de botellas de whisky, brandy y cognac, aunque con los sellos viejos y cerrados. Asimismo, sobre cada superficie se ubican detalles, portarretratos, figurillas en porcelana y cristal.

Finalmente, al lado de la entrada, una serie de platos decorativos cuelgan de la pared, algunos españoles, otros checos, con figuras talladas o pintadas en su superficie. Y justo al lado, donde se ubica una ventana de tres metros de largo por dos de alto, la totalidad de la única fuente de luz natural de la sala es mediada por una vitrina llena a tope con porcelanas Lladró, cristalería con incrustaciones, platería, decoraciones y portarretratos, mientras que a los pies de la vitrina reposan revistas y otros objetos cuidadosamente amontonados.

La sala de Lucy solo cuenta con un usuario, esta abuela de dos nietos. Podría decirse que en el transcurso de su vida ha acumulado muchas cosas para contar. De

2 La sala de Lucy se ubica en un apartamento de un segundo piso de una casa del barrio La Soledad. de la ciudad de Bogotá Ella vive sola, es madre de una hija y abuela de dos nietos. Una crisis financiera hace aproximadamente diez años la obligó a dejar una casa grande en el norte de la ciudad y trasladarse a un apartamento. En el periodo anterior a la crisis, contaba con alto poder adquisitivo. 
ahí que el espacio de la sala esté densamente poblado por múltiples objetos. Ello hace que el esquema praxológico de la sala (esto es, las opciones de movilidad e interacción determinadas por las posiciones relativas de los objetos) parezca limitado. Los objetos, prestadores de servicios por naturaleza, no tienen suficiente espacio para prestarlos, por lo que su función también sufre un desplazamiento. Los objetos pierden su función utilitaria a causa de un principio estético que se resume, en palabras de Lucy, como: "tener una sala a gusto propio". Curioso retorno del "gusto" en este siglo, que empero no remite a ese factor normativo que le atribuyera el empirismo humeano, sino a la idea de una sensibilidad individual, un deseo de confort doméstico y un placer por la distinción social. Para Lucy, su sala es un proyecto visual que provee sensaciones agradables, que ofrece una fruición emocional, en donde, al decir de Lipovetsky y Serroy, "todas las piezas de la casa son el objeto de una investigación decorativa para el placer, menos conformista que personalizado" (354) ${ }^{3}$. Para lograrlo, el espacio se dispone como una verdadera sala de exposición museal, donde, a pesar de la limitación del área, la función del objeto deviene esencialmente simbólica: está ahí no para ser usado, sino para ser visto en tanto simboliza otra cosa - una vivencia, un evento, un recuerdo, una oportunidad, etc.- que, a la vez, responde al criterio unificador de la vida de Lucy.

En la figura 1 del esquema praxológico, se muestra la limitación de desplazamientos posibles en esta sala. De hecho, Lucy no come en el comedor, y pocas veces se sienta en el sofá, solo cuando recibe visitas, lo cual no es frecuente. Los hexágonos señalan zonas de objetos que impiden la circulación. Sin embargo, aunque tiene una evidente saturación visual, la sala de Lucy no puede ser descrita, acuñando un término de moda, como la sala de un acumulador. Muy por el contrario, cada objeto de este escenario está meticulosamente ubicado y cuidado. De esta manera, si seguimos el hilo interpretativo de la sala de exposición, la sala de Lucy se muestra como un espacio museal en donde la participación del espectador responde a la "praxis" de lo observado. Adoptando la idea de la museología contemporánea de que ni espectador ni objeto de exposición son meramente pasivos, sino que uno se realiza en la performatividad del otro, diríase entonces que frente a la reducción praxológica del usuario (Lucy y sus posibles visitantes), los objetos son los protagonistas y responsables de una serie de acciones que se hacen visibles en su contemplación. Para Michel de Certeau, situaciones como esta muestran que el consumo siempre deja un margen de escape al condicionamiento de la producción. Siempre hay una "manera de hacer" que se manifiesta en el uso que se hace del objeto consumido y que refleja la "manera de ser" del usuario.

Estas "maneras de hacer" constituyen las mil prácticas a través de las cuales los usuarios se reapropian del espacio organizado por los técnicos de la producción sociocultural. Plantean cuestiones análogas y contrarias a las que abordaba el 
FIGURA 1

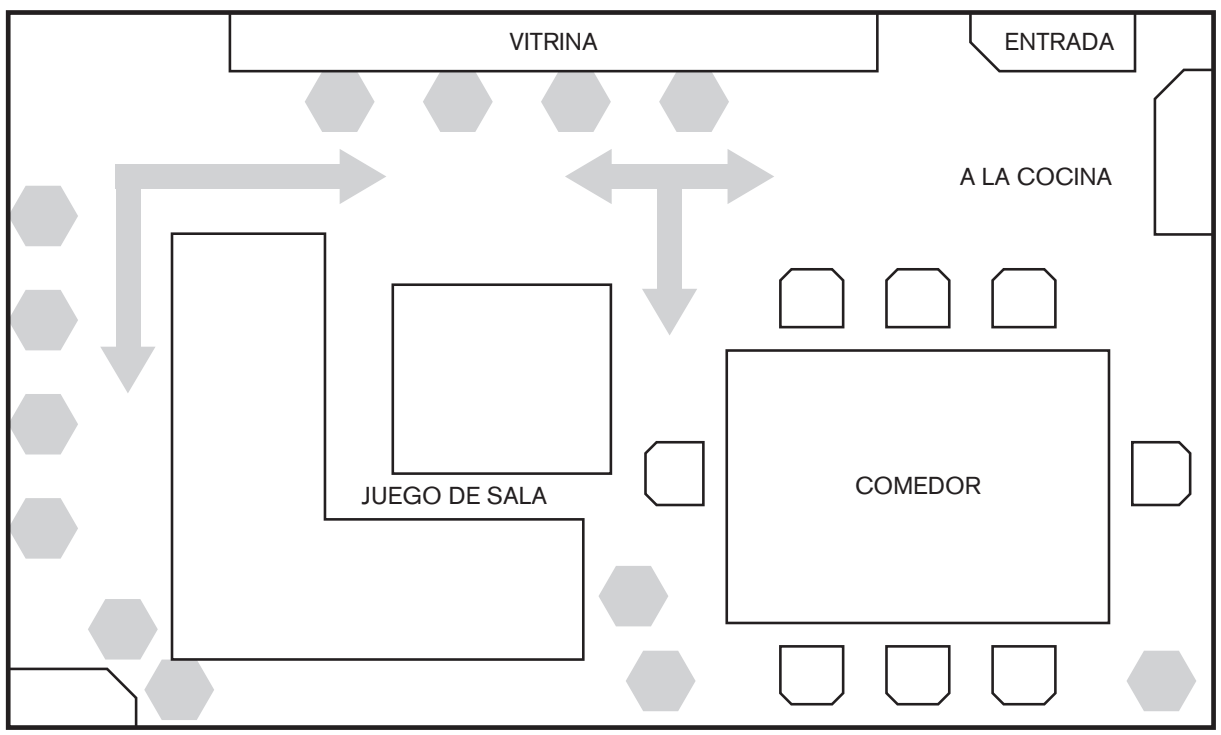

Esquema praxológico de la sala de Lucy.

libro de Foucault: análogas, pues se trata de distinguir las operaciones cuasi microbianas que proliferan en el interior de las estructuras tecnocráticas y de modificar su funcionamiento mediante una multitud de "tácticas" articuladas con base en los "detalles" de lo cotidiano; contrarias, pues ya no se trata de precisar cómo la violencia del orden se transforma en tecnología disciplinaria, sino de exhumar las formas subrepticias que adquiere la creatividad dispersa, táctica y artesanal de grupos o individuos atrapados en lo sucesivo dentro de las redes de la "vigilancia" (De Certeau XLIv).

Los objetos de la sala de Lucy son productos de consumo, y la mayoría ha sido adquirida en diversos lugares del mundo. La capacidad adquisitiva que poseía Lucy le permitió adquirirlos en el extranjero y traerlos al país hace más de treinta años. Pero una crisis financiera hace diez años impidió continuar este tipo de consumo y la obligó a trasladarse de una casa grande a un apartamento. Muchos objetos de la casa permanecen actualmente en una bodega, por lo que en el apartamento se expone una selección realizada a partir del "gusto propio" y basada en materiales, colores, marcas, hechuras y, sobre todo, en el simbolismo implicado en cada objeto. Esto indica que en esta sala se hace uso del consumo para generar una praxis a partir de los objetos visibilizados. Esta praxis, como veremos, coordina las connotaciones simbólicas ligadas a la distinción social y el valor representativo de lo europeo - un valor colonizador que impactó las clases ociosas en Latinoamérica e influyó en la estilización del entorno doméstico, según relata Morales (118)-, así como al deseo de 
FIGURA 2

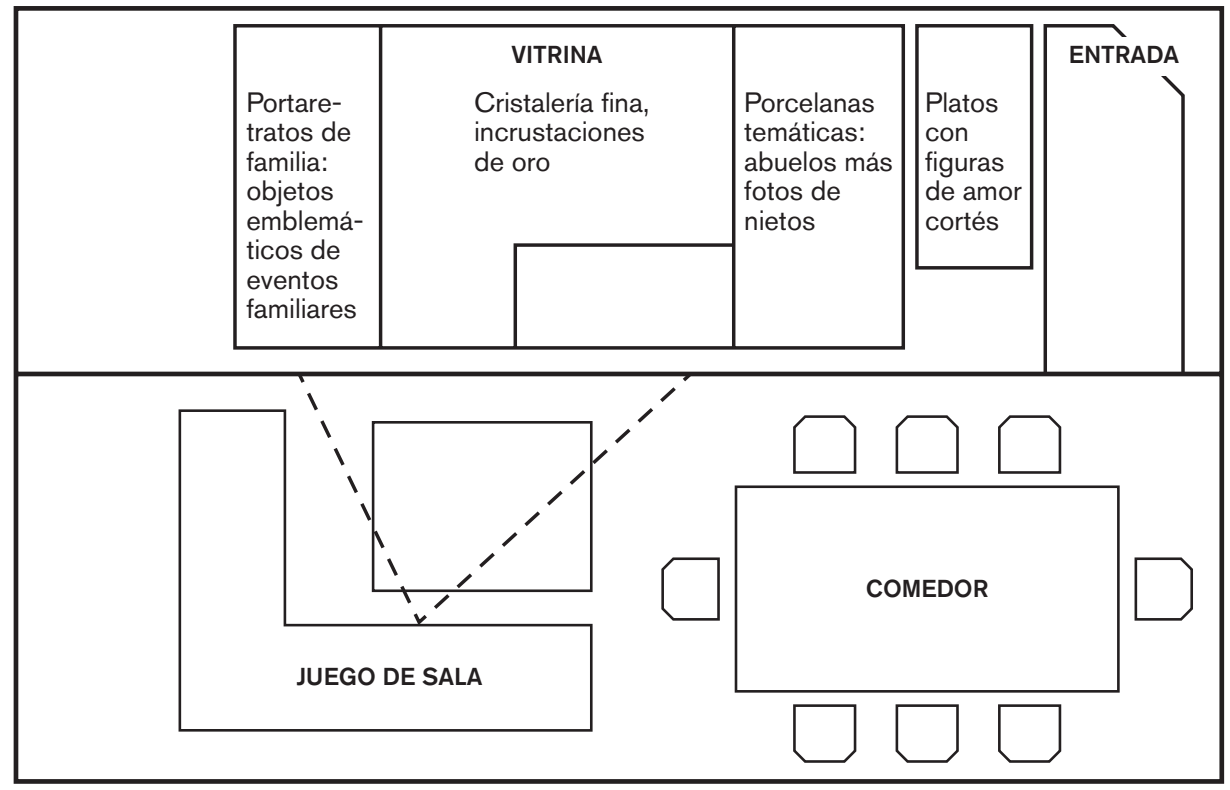

Narrativas visuales en vitrina.

singularidad de la propia vida que los objetos expuestos (bajo su uso de exposición) deben materializar y comunicar ${ }^{4}$.

¿Cómo visualizar la performatividad de este contexto objetal? Iniciemos con el caso más evidente: la vitrina. Como se mencionó más arriba, la vitrina está sobrepuesta en la ubicación de la ventana de la sala. Debido a su amplia dimensión, permite distribuir los objetos en tres secciones que dan cuenta de al menos cuatro narrativas distintas, tal como lo ilustra la figura 2.

En esta figura, la mitad superior corresponde al espacio de la vitrina, y la pared respectiva y la inferior al espacio de la sala. En la sección derecha de la vitrina se encuentra una serie de porcelanas Lladró. Cuenta Lucy que cuando fueron adquiridas, en su mayoría hace más de veinte años, tener una porcelana de este famoso taller constituía un lujo exclusivo. Además, la delicadeza de la textura y formas es signo de "ternura" y "elegancia", según sus propias palabras. Pero recordando que lo visibilizado

4 Sobre la relación entre objeto y comunicación, Méndez señala: "Sea cual sea la forma en que consumimos, todo responde a un concepto: comunicación. Tomamos cierto tipo de bebida para comunicar cierto tipo de mensaje. No es lo mismo tomar té con la tía, que tomar cerveza con los amigos, ni tomar whisky con el jefe. No es lo mismo tomar vino de caja que vino de cosecha 1980. Este tipo de comunicación está inscrito dentro de los patrones culturales que constituyen la realidad y por tanto la vida cotidiana de una cultura en particular. Las personas consumen con una intención comunicativa. Los objetos que el individuo consume «son en su mayor parte, formas de conseguir objetivos y no objetivos en sí mismos». Los objetos son una forma de mostrar lo que se es frente a lo que no se es, creando una definición propia basada en la diferencia" (298). 
FIGURA 3

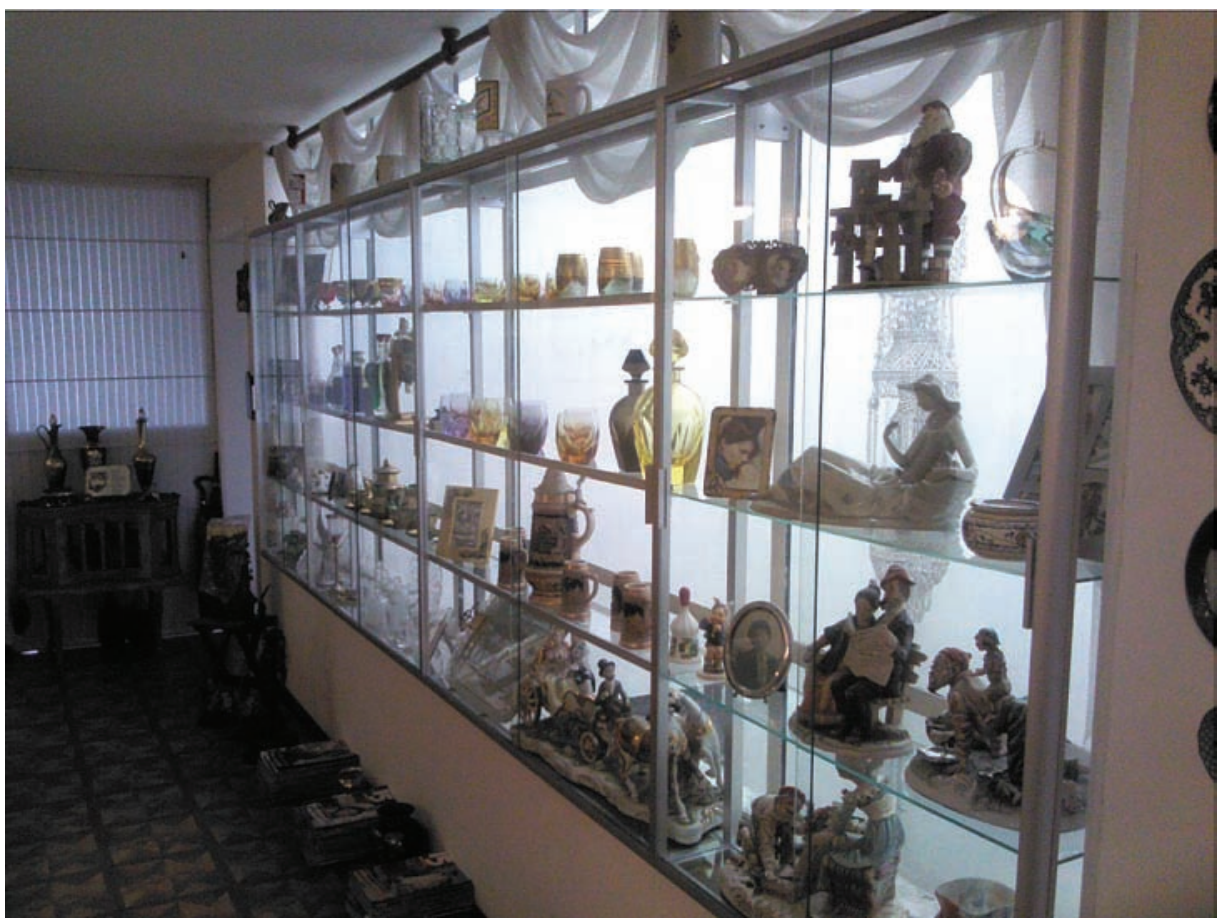

Vitrina de Lucy.

en esta vitrina obedece a un reciente ejercicio de selección de objetos, llama la atención también el vínculo temático de las figuras representadas. Resaltan particularmente tres íconos de abuelos, pues junto a las porcelanas se ubican dos portarretratos de su primer nieto y su única hija. De esta manera, como se observa en la siguiente fotografía (fig. 3), el simbolismo de la marca y el lujo de la pieza son resemantizados en una narrativa ${ }^{5}$ visual del valor de su propia identidad como abuela; el valor simbólico del lujo es usado para resaltar el valor simbólico de su identidad en el árbol familiar.

En la sección izquierda se encuentran portarretratos familiares, tanto del matrimonio de su hija como de sus hermanas, junto a objetos emblemáticos de acontecimientos personales. Si bien los objetos no son dispuestos por un hilo temporal, cada uno evoca un evento especial. Por ejemplo, se encuentra una copa de la primera

5 El concepto de narrativa es tomado aquí de Ochs y Capps, quienes presentan la siguiente definición: es el encuadre verbal, visual o corporal de una secuencia de eventos vitales actuales o posibles (19). Esta definición aplica a todas las maneras de narrar, ya sean escritas, visuales, plásticas, sonoras o corporales. Lo que resulta clave de la definición es qué entender por "eventos vitales", a saber, aquellas experiencias vividas como un drama social. Eventos o cambios significativos en el orden de la vida activa pueden generar un drama en el sentido de afectar el sentido de vida que la persona sostenía antes del evento o cambio. La flexibilidad de los medios para exponer la narrativa, puesta en relación con los contextos objetales, nos permiten diferenciar entre un modo temporal de narrar el drama correlativo a un orden histórico biográfico y un modo espacial correlativo a la exposición de un momento de relativa duración en la dimensión biográfica del contexto objetal. 
visita con la hija a Madrid, cuando asistieron a ver El lago de los cisnes hace treinta y cinco años; y a su lado, el pequeño vaso que conmemora la participación de la hija en el primer evento del Minuto de Dios en Colombia, donde solía asistir la clase alta de la capital y se realizaban sustanciosos donativos; pero también se exponen objetos preciados, como un huevo decorativo con incrustaciones que adquirió en un viaje a Europa con su exesposo. Así, la ausencia de un hilo temporal no excluye la composición de una narrativa relativa a la conmemoración de eventos biográficos personales y de su vida familiar.

Llama la atención que estos dos extremos sean los márgenes de lo visible en la sección central. Como si el simbolismo familiar contuviera los significados de esta nueva sección, compuesta por cristalería fina. Sin embargo, a la vez, su posición céntrica en la fuente de luz natural y su materia transparente se conjugan para generar un efecto de realce sobre todo lo anterior; la luz atraviesa los cristales para exaltar su presencia. En este caso, la cristalería proviene de Checoslovaquia (hoy República Checa): botellas de colores y juegos de vasos con incrustaciones en oro. En particular, para Lucy, la presencia del metal noble guarda gran valía. El oro es símbolo de riqueza, y usado de esta manera sirve para ostentar una capacidad adquisitiva y la pertenencia a una clase social. Regresa aquí el papel del consumo ostentoso como un signo de distinción social (Baudrillard); sin embargo, el consumo comporta también una función mnémica, pues en el contexto objetal específico recuerda a Lucy los años en que podía contar con lujos como estos. Este consumo ostentoso se refuerza con otro microcontexto disponible en la sala, a saber, el compuesto por los dos bares de madera venecianos, la mesa de centro y el comedor mismo. Cada bar fue traído en barco desde Venecia, de modo que su relato de origen hace parte de su valor ostensivo. Pero llama la atención el papel del cristal: junto a los dispuestos en la vitrina, los bares son altares de cristales de licor, y la mesa del comedor está elaborada con un costoso vidrio de una pulgada de ancho. La preferencia y asociación simbólica del material terminan por distribuirse a lo largo de la sala marcando un territorio por efecto del consumo ostentoso disponible a la vista.

Ahora bien, frente a la vitrina se compone otro escenario. Este ya no está elaborado por los detalles de pequeños objetos, sino por lo monumental: el comedor ya mencionado, pero también los sendos sofás y, sobre todo, la imponente mesa de centro que rodean los sofás. Estos tres tipos de objetos ocupan la mayor parte del área de la sala. Pero precisamente su apariencia monumental genera un efecto de mutuo realce entre los detalles de la vitrina y la imponencia de los muebles. Con ello, se genera el escenario donde los objetos de la vitrina son espectáculo para el sofá. El sofá localiza el punto donde todas las narrativas visuales descritas en la vitrina se hacen plenamente visibles; lo cual, a su vez, lo convierte en centro del territorio conformado por este contexto objetal.

Finalmente, las relaciones entre los objetos muestran dos “actos de visión" apelando a la expresión que Mieke Bal acuña en base a la teoría de los actos de habla 
de John Searle ${ }^{6}$ - que delimitan el territorio. Por una parte, Lucy endosa a una serie de objetos la proveniencia foránea que exalta su valor simbólico y que en conjunto demarcan una línea compuesta por: el biombo español, la mesa hindú, el baúl canadiense, el bar veneciano, los objetos extranjeros de la vitrina y los platos españoles. Esta serie de objetos hace visible el valor de lo foráneo en la historia vivida por Lucy, testimonian sus viajes y relaciones sociales de clase, exaltan el consumo ostentoso y circundan la atmósfera general de este contexto objetal. Pero en sentido estricto, solo delimitan la mitad de esta atmósfera, pues, por otra parte, reaparece en el otro costado de la sala un acto de visión ligado a la pertenencia a una localidad. Este acto de visión se muestra como una representación de lo local: está compuesto por los bodegones de frutas tropicales, la palmera de bronce, las plantas artificiales de especímenes regionales, un sol de forja y una litografía de Diego Rivera de una niña campesina. En conjunto, indican una imagen de lo local, más bien tropical y rural, del lugar de pertenencia de Lucy, que contrasta con la frontera opuesta de lo foráneo. Así, entre lo extranjero y lo local, estas fronteras brindan clausura a la exposición del espacio museal cuyo criterio curatorial yace en la historia de vida y en los procesos de identificación fomentados por los objetos de la sala: identidad de abuela, éxitos personales y familiares, distinción de clase por consumo ostentoso y traslación permanente entre lo foráneo y lo local. La tensión y complementariedad entre las fronteras de lo foráneo y lo local engloban las narrativas afectivas, biográficas, familiares y sociales que se disponen en el escenario. Estas a su vez, de manera particular y en conjunto, actúan poniendo en relación los actos de visión, con lo cual generan varios procesos de identificación (cuya exposición ocurre incluso con independencia de la presencia circunstancial del usuario). Los objetos son por sí mismos actores de la narrativa y su identificación: la componen, la simbolizan y la comunican.

6 Los estudios visuales, y en particular Mieke Bal, han expuesto que la visibilidad consiste en un "uso retórico de la materialidad" que produce lo visible al interior de prácticas y regímenes discursivos cultural e ideológicamente vigentes. Como afirma Bal: "si la lectura de los objetos incluye a lo social, a la gente, su estudio incluiría también a las prácticas visuales posibles dentro de una cultura particular y, por tanto, dentro de regímenes escópicos o visuales; incluiría, en definitiva, a toda forma de visualidad" (15). Así, un acto de visión es una construcción pragmática de significación mediante el uso de elementos semánticos para la consolidación y comunicación de un mensaje suplementario. Pero a diferencia de los actos de habla de Searle, en los actos de visión el contexto, siguiendo la crítica derridiana a Searle, modifica el sentido de lo visto de una manera más efectiva que la intención. De lo contrario, sería imposible atender y recibir el mensaje inscrito en un contexto objetal sin conocer la intención del usuario; pero precisamente las narrativas visuales, la naturaleza sígnica del objeto de uso y la materialización sensible de procesos de identificación en el contexto objetal dan cuenta de que el conjunto final configura una estructura significante que sobrepasa las intenciones del usuario. De ahí que, a causa de las significaciones y valores culturales inscritos en la práctica de sociabilidad, en la mediación generada por los objetos de uso entre lo designado en la producción y distribución y lo apropiado, así como en la consolidación de un efecto pragmático de sentido que tiende a unificar el espacio "sala-de- $\mathrm{x}$ " puedan producirse modos distintos de visibilización a través del acto de visión compuesto en dicha estructura significante. Lo visto es constituido, performativamente, por el acto de visión resultante del acto re-creativo del usuario que, empero, el todo final supera con creces. 


\section{La performatividad de los objetos}

¿Por qué hacer de la sala un espacio museal que dispone tal variedad de narrativas y procesos de identificación, un escenario performativo correlacionado que se hace hábitat? El habitar en la sala de Lucy está marcado por la distancia temporal. Recordemos que la crisis financiera personal de hace diez años puso fin a las prácticas de consumo ostentoso y que en el apartamento donde vive hoy se aplicó un esfuerzo de selección de los objetos que actualmente la acompañan. Pero todos los objetos de la sala pertenecen al periodo precrisis. Por ello, este contexto objetal no es meramente biográfico sino conmemorativo, en el sentido de conservación de un pasado perdido. La sala se compone de objetos que testimonian, conmemoran y hacen visible una distancia temporal y un presente consecuente: función tradicional del espacio museal. Pero al mismo tiempo, cada objeto juega un papel performativo en la pragmática de hacer visible: ejerce la comunicación, mientras que el criterio de selección y exposición de dichos objetos hace "obra abierta", una totalidad narrativa abierta y suficiente que se crea en la interpretación, por demás, indeterminada.

Al hacer este ejercicio, la sala de Lucy también se convierte en un lugar de refugio y recogimiento contra el tiempo; o, en palabras de Cassigoli, la sala se hace "morada", esto es, "el intento más o menos consciente, más o menos programado, de retener el paso tumultuoso del tiempo de 'allá afuera', la fuga heracliteana de las cosas que envejecen, que se desvencijan, que se marchitan, que se apolillan, que se van... En fin, refugio contra la contingencia y la temporalidad" (17). Poco importa la mezcla de tipos de objetos heterogéneos frente a esta construcción de morada como práctica de habitar; lo relevante es que esa morada sea el acontecimiento central de la sala donde el paso del tiempo es repudiado. Así, las tensiones entre lo foráneo y lo local, lo íntimo y lo social, lo monumental y el detalle, lo ostentoso y lo biográfico inmovilizan el tiempo, o mejor, lo espacializan para lograr que lo heterogéneo conviva en una misma y barroca atmósfera sensorial que favorece las identificaciones de Lucy y su necesidad de pertenencia al mundo. "Esta es la particularidad del barroco: mantener juntos elementos perfectamente heterogéneos. [...] Ciertamente es posible poseer cosas, pero poco a poco uno se ve poseído por ellas. Y es esta 'posesión', común para la gran mayoría, lo que funda, de manera detallada, la comunidad humana" (Maffesoli 168). Sin embargo, por barroca que sea, la sala de Lucy es el vínculo que, mediante su permanente pragmática, une a esta usuaria con su imagen del mundo y de sí misma.

Lo anterior nos introduce en la performatividad de los objetos en virtud de la relación coconstructiva entre el/la usuario(a) y los mismos objetos. Esta relación está marcada por una estética lúdica, dinámica, que permite y ratifica la identificación de Lucy con el escenario objetal. En palabras de Maffesoli, "el objeto se hace lúdico con el simbolismo", es "consumado" reiteradamente en el juego estético de despertar emociones y convocar una comunión (225). El efecto de este juego, siguiendo a este 
teórico de la cultura, es la generación de un escenario semiótico donde lo individual y lo colectivo, la memoria y la proyección se ponen en relación para hacer circular emociones, afectos y símbolos, en suma, producir una estética. Maffesoli denomina este efecto "teatralidad". El escenario teatral se diseña y compone para dar lugar a la actividad, de modo que cosas humanas y no humanas puedan interactuar con el fin de incitar una fruición estética comunicable. La teatralidad puede estar compuesta por una narrativa temporal que haga visible una historia o armada como un rompecabezas espacial con la intención de hacer común elementos heterogéneos. Pero, en cualquier caso, expresa siempre el vínculo entre lo público y lo privado, lo individual y lo colectivo, lo subjetivo y lo intersubjetivo en la sociabilidad de un lugar para ser leído y respecto del cual el usuario lleva a cabo su identificación: "volens nolens, coexisto en un conjunto donde todo hace cuerpo: coexisto por supuesto con los demás que me constituyen por lo que soy, pero coexisto también con esta multiplicidad de objetos sin los cuales la existencia contemporánea es ya inconcebible" (Maffesoli 213). Esta coexistencia no es meramente pasiva. De hecho, para que sea posible entender la propia existencia a partir de los objetos con que construimos nuestra morada, se requiere de una acción, de una permanente actividad creativa en el orden de lo visual que vincule la pulsión, la significación, la creencia, la incertidumbre, la rutina, a sí mismo y a otros, en suma, la vida inmediata y cotidiana en el uso del objeto, las prácticas a las que sirve y las representaciones que moldea. Esto es lo que Lipovetsky y Serroy entienden como la posibilidad de "agenciar un entorno creativo e imaginativo, dar un rostro singular a la casa donde uno quiere sentirse bien":

El valor estético ha superado el valor estatutario tradicional: al conformismo burgués sigue un individualismo decorativo marcado por la afirmación de los gustos subjetivos, la pluralidad y el eclecticismo estético. Desacralización del mueble, deslegitimación de la impersonalidad de la decoración, búsqueda de ambiente, vestimenta personal de los espacios de vida, primado del placer y la singularidad decorativa: estamos en el momento de la psicologización, de la afectivización de la relación estética con la casa (355).

De esta manera, la teatralidad conlleva la posibilidad de realizar un acto de re-creación de la propia vivienda, de la propia casa y, en consecuencia, de la vida cotidiana. Este ejercicio, que caracteriza la performatividad de los objetos, puede ser comprendido desde dos puntos de vista simultáneos e ineludibles. Por una parte, dicha recreación reproduce los desarrollos del capitalismo en los siglos xx y xxI. Según Lipovetsky, junto con el fortalecimiento del desarrollo globalizado del modelo económico-político moderno, el capitalismo ha terminado por diluir el límite otrora aparentemente inconmovible entre lo económico y lo estético. No solo porque el arte hoy se mueve como una gran empresa con un comercio especializado, sino porque lo económico ha integrado una dimensión estético-emocional central en la competencia mercantil. Publicidad, mass-media, mercadotecnia, diseño, moda, plataformas y centros comer- 
ciales movilizan afectos y emociones como parte de la distinción de los productos, la estilización de los bienes y de la generación de hábitos de consumo. A esto denomina Lipovetsky "capitalismo artista" o "creatividad transestética" (12). Esto significa que, junto con la superabundancia de objetos de consumo, se genera una inflación estética que se infiltra "en todos los intersticios del comercio y la vida ordinaria", un "hiper-arte" que implica la "generalización de estrategias estéticas para el mercado en todos los sectores de las industrias del consumo" y que, para el consumidor, implica el consumo de estilos, de sensaciones y experiencias sensibles y de una actitud estética hacia la vida (26-30). La posibilidad de crear una atmósfera, de configurar un contexto objetal dinámico y modificable que pueda ser elemento de (auto)identificación, de jugar con los detalles y convertirlos en signos de actualidad constituye una apropiación reproductiva de este funcionamiento estético del capitalismo artista, según el cual el consumo y lo consumido se perciben y hacen ver una afirmación estética personal de un individuo sobre la propia vida.

Por otra parte, frente al flujo del capitalismo artista que atraviesa la composición del contexto objetal "sala", el uso de los objetos enseña que, en paralelo a la faceta reproductiva de la recreación, existe también una faceta "laboral" o "artesanal" de la misma. En términos generales, la artesanía es un modo de producción de objetos centrado en la reproducción no de los objetos mismos (como en la producción industrial), sino del valor o significado cultural transmitido en su elaboración. La sapiencia y creatividad del artesano se halla en la habilidad para dialogar con la materia a fin de perfeccionar la expresión de una tradición; de ahí que cuando no hay una generación que dé continuidad a la tradición, ese saber tienda a desaparecer. Sennett retoma esta estructura básica de la artesanía para señalar que aun en la sociedad industrial existe la artesanía como un modo de vida; para él, "artesanía designa un impulso humano duradero y básico, el deseo de realizar bien una tarea, sin más [...] es aplicable al programador informático, al médico y al artista; el ejercicio de la paternidad, entendida como cuidado y atención de los hijos, mejora cuando se practica como oficio cualificado, lo mismo que la ciudadanía" (20). Más de una faceta de la vida demanda la atención del oficio artesanal, del sostenimiento de la tarea, la acomodación de los elementos y la permanente actitud de hacerlo bien -y, en consecuencia, la apertura permanente al posible fracaso y corrección del curso de acción que permiten concebir esa actividad como un inacabable proyecto creativo. Configurar un contexto objetal implica esa relación entre pensamiento y habilidad que "se advierte en dominios aparentemente tan distintos como la albañilería, la cocina, el diseño de un patio de recreo o la ejecución musical al violonchelo" (21). La energía emocional y cognitiva necesaria para sostener la sala de Lucy como un lugar de conservación y aprendizaje identitario implica atender y componer permanentemente el contexto objetal para dicho fin; el contexto objetal de la sala debe ser flexible y abierto al uso posible y, más aun, a la llegada de nuevos objetos producto de eventos, festividades y regalos familiares. 
En virtud de estas dos facetas recreativas de un contexto objetal, podríamos decir que la performatividad es una actividad caracterizada por poner en relación la presión cada vez más inevitable del capitalismo artista, que exalta la estetización de la vida cotidiana, con la proyección, energía y oficio de la actitud artesanal, que implica componer y sostener un contexto objetal al servicio de una actividad y en concordancia con el conjunto de significados que la legitiman. Caso contrario, los procesos de identificación no serían posibles, pues todo lo visible siempre supone "una estrategia de selección que determina qué otros aspectos o incluso objetos permanecen en la oscuridad" (Bal 18) ${ }^{7}$. Gracias a la performatividad, el objeto tiene la posibilidad de visibilizar representaciones culturales en torno a los modos como una persona construye su propia vida, los procesos de identificación que reproduce y las maneras en que los interpreta o altera. Los objetos iluminan, por esa mutua dependencia y coconstrucción con el usuario, las posibilidades experienciales mismas e inmediatas de la vida, las maneras como son interpretadas y comunicadas a otros. Esto significa que los objetos hacen visibles las correlaciones entre las esferas de vida y la producción de un individuo estético que se ocupa de su existencia y que construye procesos de identificación también en la diferencia con los objetos y lo que ellos pueden visibilizar; hacen ver una estética autorreflexiva fundada en la inquietud de sí para con otros, de cómo ser y ser visto por otros. En suma, revelan cómo la experiencia relativiza al sujeto: carente de una identidad sustancial, se da a la búsqueda inextinguible de una identificación mediante, entre otras formas, la conformación de actos de visión que puedan ser sentidos y comunicados desde el interior de su vida privada y rutinaria.

De esta manera, la performatividad de los objetos, ubicada en el intersticio de las dos facetas de la recreación del propio contexto objetal, nos plantea un último reto. En cuanto a Lucy, ¿cómo la performatividad hace posible procesos de identificación entre la usuaria y los objetos? Por una parte, hay una razón vivencial relativa a la sensación de continuidad identitaria:

\footnotetext{
Al despertar cada mañana se consuma un hecho identitario original. Reencontrar la realidad circundante como se la dejó la noche anterior y los objetos en el mismo orden, confirman que "la vida no es sueño..."; aseguran una pacífica "sustantividad resguardadora de nuestro reposo". La sensación de ser la misma persona representa la experiencia básica del yo: la identidad personal se explica esencialmente en términos de memoria, de lo recordado y de lo recordable. La frágil identidad personal depende de que el orden de los propios dominios no se trastorne de la noche a la mañana (Cassigoli 96).
}

7 Becker describe el asunto de la siguiente manera: "Sorting through the relationship between vision and knowledge leads inevitably to the relationship between the seer and the seen. What happens when people look? Seeing, like other aspects of knowledge, is not limited to cognition, but is performed in the act of looking. [...] Considering the relationship between the seer and that which is seen as a performance is inconsistent with visual essentialism. A performance perspective has the advantage of fore fronting visuality as it is being investigated, without determining in advance the object of vision or its qualities" (154). 
Pero también hay una razón subyacente a la configuración misma de la identidad subjetiva. Cuando hablamos de "procesos de identificación", apelamos a un distanciamiento de una lógica de la identidad, a fin de reconocer la multiplicidad de identificaciones que subyacen y forman a la persona. En lugar de esencias, "la lógica de la identificación pone en el escenario a 'personas' con máscaras variables, tributarias del o de los tótem emblemáticos con los cuales se identifican” (Maffesoli 16). De ahí la importancia de la performatividad en los actos de visión que componen un contexto objetal, pues si seguimos esta lógica de la identificación, la naturaleza performativa de los actos de visión no son independientes de la performatividad del acto identificatorio mismo. Cuando en el espacio museal de la sala de Lucy se consolida una atmósfera que es capaz de "hablar" por sí misma, no se está separando esta performatividad de la identificación; por el contrario, es la performatividad de la identificación la que proyecta la comunicación encarnada en el escenario completo, de manera que lo hace aparecer como autosuficiente: es el poder del simbolismo el que lo hace emblemático. La identificación requiere el esfuerzo de su producción, requiere ser sostenida y recreada -por ello mismo, es inacabable-.

Ahora bien, lo anterior no es una mera constatación de la relación entre espacio e identidad, claramente insatisfactoria cuando se agrega la performatividad del contexto objetal. Ciertamente, para la construcción del espacio, la narrativa de la vida familiar es un signo que cumple una función similar a la de lo foráneo en la sala de Lucy: ambos ponen en juego significaciones que vinculan lo público con lo privado, el pasado con el presente. Pero la performatividad obliga considerar el uso singular, la singularidad específica que es construida con esas significaciones. De lo contrario, la performatividad se reduciría a una reproducción mecánica de significados normalizados. Lo performativo se caracteriza por que junto a la reproducción siempre emerge un excedente de significación generado por el conflicto con lo reproducido; por el hecho de que, en términos butlerianos, la "citación" del enunciado performativo es tanto reiteración de la norma como alteridad de lo repetido; lo repetido es ya otro signo que no puede garantizar la determinación del performativo, esto es, la variabilidad del contexto del que depende el performativo conlleva siempre la posibilidad de lograr un uso desviado de lo repetido (Boccardi 28). De esta manera, Butler nos recuerda que en la performatividad se requiere ciertamente la reproducción de normas; de lo contrario, carecería de inteligibilidad, esto es, de "legibilidad en el espacio social y el tiempo, así como una relación implícita hacia los otros [...] que está condicionada y mediada por normas sociales" (Butler, "Performatividad, precariedad" 333). No obstante, esas mismas normas entran en crisis al ser reelaboradas en la performatividad misma. El conflicto que esto genera es fuente tanto de inclusión como de exclusión, en particular, de procesos de identificación social culturalmente legitimados. "La performatividad tiene completamente que ver con 'quién' puede ser producido como un sujeto reconocible, un sujeto que está viviendo, cuya vida vale la pena proteger y cuya vida, cuando se pierde, vale la pena añorar" (335). Otra manera de decirlo es 
que lo que escapa a la crítica de Baudrillard al consumo ostentoso es esta condición fundamental, este vínculo íntimo entre lo que el objeto significa como lujo y lo que "hace" para hacer visible una identificación que une a la persona con otros y con el mundo: lo que hace el bar veneciano no solo es ostentar un lujo, sino esperar una comunión social que permita rememorar lo que era Lucy y dar fe de lo que es ahora.

Por ello, cuando Lucy realiza el esfuerzo de identificarse, en el acto performativo mismo, con una teatralidad de historia de vida, de hecho, está actuando sobre sí misma. O, mejor, efectúa un escenario que "habla" de ella, es decir, la constituye subjetivamente. Los actos de visión tienen un carácter performativo en la construcción de un contexto objetal que responde a la práctica cultural de habitar, de generar un hábitat personalizado. Pero esa misma performatividad está en el orden del significante, cuyo significado es construido en el proceso de identificación del usuario. Ello es lo que alienta la necesidad permanente de renovar el repertorio de la sala, de renovar la identificación con el contexto objetal; como cualquier otro lenguaje, no puede acabar. De esto se sigue que lo que habita una sala es un sujeto en composición, un sujeto que encuentra en su hábitat una interpelación a ser de determinada manera. En esta línea, Butler afirma:

El performativo no es meramente un acto usado por un sujeto pre-dado, sino es una de las poderosas e insidiosas maneras por las cuales los sujetos son convocados como seres sociales, inaugurados en la socialidad por una variedad de interpelaciones difusas y poderosas. En este sentido, el performativo social es una parte crucial no solo de la formación de sujetos, sino de la continua lucha y reformulación política del sujeto. En este sentido, el performativo no solo es una práctica ritual: es uno de los rituales influyentes por los cuales los sujetos son formados y reformulados ("Performativity’s social magic" 125).

Diríamos entonces que la performatividad tiene una doble faz: la del acto de visión, la narrativa visual construida por las relaciones entre objetos, y la del proceso de identificación, formador y reformulador de subjetividad ${ }^{8}$. Pero aún queda una cuestión: ¿qué hace que el habitar de Lucy sea diferente del habitar de otro? ¿Por qué ejercen performatividades distintas? Esta cuestión es crucial cuando se recoge el papel de la interpelación involucrado por Butler en la última cita ${ }^{9}$. En efecto, el territorio

8 Al respecto, Maffesoli afirma: "De esta manera el medio, en tanto que mezcla, sería la condición de posibilidad de la existencia humana a partir de la existencia social y de la existencia natural. Lo que significa que el 'yo no puede tomar conciencia de sí mismo' más que en relación. En esta perspectiva hay una forma de acomodo en su sentido óptico, pero acomodo en los dos sentidos: por una parte, me acostumbro a ver lo que me rodea y a los otros que comparten este entorno, y por otra parte yo soy visto por la alteridad física y social. Doble movimiento que me constituye en lo que yo soy. Es esta doble reinversión del medio la que confirma el orden estético. El primum relationis que es causa y efecto de dicho orden y por ende del de la relación interpersonal, pero igualmente el de la relación con el entorno físico, con este 'dado' que constituye el sitio donde yo vivo" (198).

9 Butler afirma: "How would one distinguish -in practice- between the social and the linguistic on the occasion of that ritual of social inauguration and maintenance by which a subject is alerted to its 'place' through the name it is called or a subject is formed through the name that it understands itself to be called without there having been an official call? If the habitus is both formed and forming, and if such interpellations are central to both that 
conformado por el consumo ostentoso en la sala de Lucy reproduce interpelaciones ideológicas ligadas a la distinción social y a la pertenencia de clase. Tal consumo demanda una forma de ser y la valoración del lujo y del poder adquisitivo, así como establece una distancia entre quien lo posee y quien no; los objetos demuestran "ser de una clase" social. Por ello, frente a la frustración práctica poscrisis, se expone la saturación del contexto objetal que la interpela a ser todavía como entonces.

La performatividad, empero, no acaba en la interpelación. Junto con la citación de las normas, aparecen una serie de acciones que modulan la manera como cada sujeto personaliza su práctica de habitar, lo que equivale a la manera como promueve procesos de identificación en la performatividad de su propia subjetividad. A estas acciones Michel de Certeau denomina “tácticas". Según este autor, las tácticas se oponen a las estrategias, entendidas estas como el cálculo de relaciones de fuerzas que aíslan la posición de un sujeto en un ambiente. A tal respecto, la arquitectura sería esencialmente estratégica. Por ejemplo, el modelo de vivienda en construcción vertical, según Birdwell y Lawrence, supone una estrategia discursiva de distribución de la población que, a su vez, se corresponde y valoriza (monetaria y simbólicamente) en función de las relaciones de proximidad con las zonas de poder comercial y político de la ciudad (28). Lucy, así como muchos otros, recibe una estructura de vivienda conforme a esta lógica, en tanto residente de un apartamento -y, asimismo, las interpelaciones respectivas constituyentes del contexto objetal también moldean este registro estratégico de la vida cotidiana-. Pero frente a la estrategia, la táctica utiliza lo que ofrecen las relaciones de fuerza para generar una singularidad en el presente. La táctica es, para de Certeau, la habilidad del débil para redirigir las fuerzas en "maneras de hacer" lo cotidiano, de tal forma que él se presente como fuerte (44).

La performatividad del usuario para hacer un hábitat correspondería a una táctica. El habitar inscrito en la sala de Lucy se distingue por una visualización espacial de un tiempo pasado que en el presente interpela la acción e imagen de sí misma, todo ello mediante las disposiciones, selecciones y ordenamientos de los objetos en un espacio de exposición que recuerda día a día su identificación. Es una táctica de reconstrucción permanente de sí.

Las tácticas son procedimientos que valen por la pertinencia que dan al tiempo: en las circunstancias que el instante preciso de una intervención transforma en situación favorable, en la rapidez de movimientos que cambian la organización del espacio, en las relaciones entre momentos sucesivos de una “jugarreta”, en los cruzamientos posibles de duraciones y de ritmos heterogéneos, etcétera (De Certeau 45).

De esta manera, la subjetividad se ve enmarcada por dos tipos de poder que constituyen las condiciones de posibilidad del consumo, la apropiación y los procesos de

formation and its formative effects, then social interpellations will be performatives on the order of the habitus, and their effects will be neither linguistic nor social, but indistinguishably -and forcefully- both" (126). 
identificación en el seno mismo de la performatividad. El poder estratégico que ordena, coordina y delimita la práctica del habitar, y que antecede la praxis del usuario; y el poder táctico que se implementa en la performatividad y que tiene lugar en el uso que hace de los elementos ofrecidos por lo estratégico. Ambos, entonces, corresponderían respectivamente a la faceta de lo político y de lo estético en el mundo objetal, tal como veremos a continuación.

\section{Estética y política del mundo objetal}

El contexto objetal es reunión y expresión de conflictos, contradicciones y acuerdos -tensiones, en general- en el orden de las representaciones culturales; estas, a su vez, se entremezclan en las prácticas sociales que media cada contexto objetal. Así, la performatividad del contexto objetal remite a las tensiones, vínculos y distancias que entran en juego en su constitución. Es posible identificar al menos tres clases de tensiones. La primera se refiere al orden de la posibilidad. La sala de Lucy pone de manifiesto el encuentro entre el orden de la posibilidad y la restricción estructural. Esta sala de apartamento es diseñada arquitectónicamente bajo una estructura que, ceteris paribus, es convencional de este tipo de vivienda; además, contiene los objetos protocolares de rigor que distribuyen las actividades comunes a tener lugar. Sin embargo, la confrontación entre el número y función de los objetos y el simbolismo y narrativas que componen demanda que sobre esa estructura se module la distribución de los objetos para incorporar signos de la imagen personal de la usuaria. Esto indica que en la flexibilidad funcional de cada objeto, las barrocas relaciones interobjetales marcan el grado de posibilidad (de libertad) de interacción entre objetos y usuarios, así como entre los usuarios mismos. Para Miller, esta característica evidencia que el uso de aquellos responde tanto a un margen de restricción (de estructura) como de creatividad (de posibilidad) y que, en consecuencia, lo individual no es una muestra (microcosmos) de lo social (macrocosmos), pues lo individual, esto es, el contexto objetal de nuestra propia vivienda, presenta las mismas restricciones y posibilidades, en términos de sus relaciones constitutivas, que el marco general de lo social (13). Sin embargo, a la vez, cada caso ofrece un modo susceptible de singularidad en la manera como se establece la balanza entre lo estructural y la posibilidad.

Ahora bien, esta tensión entre posibilidad y restricción estructural se evidencia en al menos otras dos tensiones subyacentes. La primera remite al rango que se establece entre lo político y lo estético de la performatividad. Hablamos en términos de rango, pues cada caso posible de contexto objetal ilustra una manera de mediar la tensión entre estos extremos: por un lado, lo político de la performatividad recuerda el hecho de que el lugar donde habitamos es principalmente un mecanismo regulador de la conducta; el contexto objetal sala se dispone para una práctica que discrimina con cierto rigor el tipo de conducta apropiada o esperada a tener lugar, define el esquema 
praxológico posible y expone los significados y valores ideológicamente vigentes y apropiados en la propia vida cotidiana. La sala de Lucy ha mostrado esta condición en la medida en que expone expectativas de clase y consumo que, a su vez, definen expectativas sobre los usuarios y visitantes, marcan las posibilidades de acción y hacen visible la interpelación de la ostentación en la vida de la usuaria. Por otro lado, sin embargo, en esta misma sala se expone también el extremo opuesto, el estético: es precisamente por la incidencia y magnitud de la interpelación del consumo ostentoso presente en la sala de Lucy que existe una evidente y permanente fruición; los objetos ostentan porque hacen parte de un estilo de vida en construcción. La performatividad del contexto objetal juega aquí un doble papel: por una parte, desde el extremo político, genera interpelación ideológica al producir el deseo y la necesidad de apropiarse de determinados significados y valores dominantes con los cuales la usuaria realiza un proceso de identificación; pero, por otra parte, en tanto el proceso de identificación es inacabable, la performatividad de la identificación no puede sino hacer vivir esa experiencia como la construcción y mantenimiento permanente de un estilo de vida definido, esencialmente, por rasgos estéticos; esto es, por la posibilidad de concebir su propia existencia como un proyecto creativo acorde a una personalización de lo común. Sostener un estilo de vida no es, según Chaney (24), una actividad irreflexiva. Por el contrario, requiere ofrecer y sostener el sentido del conjunto de objetos, bienes, actividades y experiencias que lo diseñan. Esta es la razón por la que en la sala cada detalle es dispuesto con atención para "hablar" de su propia vida, no meramente como narración biográfica, sino como la elevación de una imagen de sí. De esta manera, el contexto objetal se evidencia también como el escenario de una tensión entre la performatividad de la interpelación mediada por la dimensión simbólica de aquel contexto y la performatividad inacabable de la búsqueda identitaria del usuario.

Esta tensión encuentra su correlato en la que ocurre entre el contextualismo del contexto objetal (valga la redundancia) y el background político y cultural del sujeto. La cuestión es que no solamente el contexto objetal presenta una faceta política por su vínculo con la interpelación, sino que el sujeto, en tanto producto de discursos y relaciones de poder (tal como ha sido ampliamente mostrado desde los análisis genealógicos de Foucault), llega a construir su vida cotidiana y su habitar con una carga política que lo autodefine en la cultura. De hecho, sin esa carga, la performatividad de la identificación no podría tener lugar, ni margen de libertad estética, pues es con base en ella que el individuo puede incorporarse en un sistema de diferencias. El sujeto reproduce las relaciones de poder y actualiza los discursos sociales en su propia existencia y, en consecuencia, en su propio hogar, en los contextos objetales que configura para habitar el mundo social. Sin embargo, así como el sujeto no es una sustancia predeterminada, el contexto objetal tampoco lo es. La performatividad que lo constituye obliga a reconocer que en la misma medida en que los actos de visión inscritos en el espacio de la sala conservan o modifican su sentido en virtud de las alteraciones del contexto de emisión, asimismo la efectividad pragmática del contexto 
objetal se modifica en función de la modificación de su contexto de realización. Esto significa que el contexto objetal está sujeto al contextualismo de la práctica cultural y de las representaciones vinculadas.

Grossberg define el contextualismo como un tipo de aproximación al estudio de la cultura según el cual "la relación precede -es más fundamental ontológicamentelos términos de la relación" (49). Precisamente, un contexto objetal no es más que relacionalidad, razón por la cual podemos extraer esta noción epistemológica para aplicarla a este caso; una relacionalidad contingente y situada cuyos efectos en la construcción de cultura varían en base a la manera como los objetos se relacionan entre sí, con el espacio y se articulan con los sujetos y su background político y cultural; articulación que genera diferencias tanto del contexto objetal como del usuario. Es por esta razón que, como indica Homi Bhabha,

los términos del compromiso cultural, ya sea antagónico o afiliativo, se producen performativamente. La representación de la diferencia no debe ser leída apresuradamente como el reflejo de rasgos étnicos o culturales ya dados en las tablas fijas de la tradición. La articulación social de la diferencia [...] es una compleja negociación en marcha que busca autorizar los híbridos culturales que emergen en momentos de transformación histórica (19).

Así, pues, el sujeto producido culturalmente llega a realizar sus prácticas con relaciones de poder y discursos dominantes, pero el contextualismo del contexto objetal abre un margen para generar otros significados y otras relaciones, margen que confronta la tradición y favorece la emergencia de diferencias en la performatividad del contexto objetal y de la identificación del usuario.

En suma, encontramos que la performatividad que atraviesa, une y coforma objetos y usuarios se consolida por los conflictos entre la posibilidad y la restricción estructural, entre la interpelación de los objetos y la estética de la identificación y entre el contextualismo del contexto objetal y el background político y cultural del sujeto. De esta manera, lo estético de la performatividad de los objetos/usuarios estaría caracterizada por el margen de posibilidad (libertad) recreativa, la fruición de los procesos de identificación y la indeterminación del contexto objetal, mientras que lo político correspondería a la reproducción de lo estructural, el rol permanente de la interpelación y el carácter político y cultural de los procesos de producción de subjetividad. Si sumamos estas condiciones, encontramos una paradójica conclusión: en virtud de la performatividad, la distinción misma entre sujetos y objetos, en términos de su constitución cultural, tiende a difuminarse. La performatividad del contexto objetal es correlativa a la de los procesos de identificación de los usuarios, de modo que el establecimiento y permanente interacción de los actos de visión para la consolidación de un mensaje viabiliza la identificación y, con ello, la dinámica de la personalidad y la reproducción de subjetividad. La permanente, fluida y productiva interacción entre lo estético y lo político de lo objetal -de la cultura material contemporánea en 
general-, a la vez que sustenta tensiones, se ha constituido en el mecanismo ideal para afirmar una construcción estética de la propia existencia en el marco reproductivo de relaciones de poder cada vez más insidiosas y sutiles. Es la abierta y constante posibilidad de introducir una ruptura en el orden de lo político lo que hace que un modo de configuración de la cultura material sea fundamental para comprender un orden social. Y en la misma medida, es en la confrontación con lo que tiene de político el contexto objetal, con la cultura material, que emerge la posibilidad de abrir campo a la divergencia del orden, a la estética y al cambio.

Miller, ya a modo de cierre, lo enuncia de la siguiente manera: no se trata meramente de que, siguiendo el clásico cuento, el emperador en realidad no tenga ropajes; se trata también de que si se eliminan los ropajes no se puede encontrar al emperador. Esto otorga potencial creativo y vital al hecho de que las posibilidades de recontextualizar el ropaje, alterar sus usos y formas en esa estética termina modificando no solo al sujeto, sino a las estructuras, prácticas y discursos que lo produjeron. En suma, entre lo político y lo estético existe una mutua retroalimentación que pervive gracias a sus tensiones fundantes: mientras lo estructural del contexto objetal reproduce la cultura y modula los comportamientos, lo posible de la estética del contexto objetal y la vitalidad de la performatividad abren siempre líneas nuevas de construcción cultural al interior de la vida cotidiana.

\section{Referencias}

Bal, Mieke. "El esencialismo visual y el objeto de los estudios visuales" Estudios Visuales 2 (2004): 11-49. Impreso.

Baudrillard, Jean. Crítica de la economía política del signo. México: Siglo XXI, 1991. Impreso.

Becker, Karin. "Where is Visual Culture in Contemporary theory of comunication?". Nordicom Review 1-2 (2004): 149-157. Web. Oct. 2013.

Benjamin, Walter. El libro de los Pasajes. Madrid: Akal, 2004. Impreso.

Bhabha, Homi. El lugar de la cultura. Buenos Aires: Manantial, 2002. Impreso.

Birdwell, Donna y Denise Lawrence. House life. Space, place and family in Europe. New York: Berg, 1999. Impreso.

Boccardi, Facundo. "La performatividad en disputa: acerca de detractores y precursores del performativo butleriano" Aesthethika 5.2 (2010): 24-30. Impreso.

Butler, Judith. "Performativity's social magic". Bourdieu: a critical reader. Ed. R. Shusterman. New Jersey: Wiley, 1999. 113-128. Impreso.

---. "Performatividad, precariedad y políticas sexuales". AIBR. Revista de Antropología Iberoamericana 4.3 (2009): 321-336. Impreso.

Cassigoli, Rossana. Morada y memoria. Barcelona: Gedisa, 2010. Impreso.

Chaney, David. Estilos de vida. Madrid: Talasa, 1996. Impreso. 
De Certeau, Michel. La invención de lo cotidiano, vol. I. Artes de Hacer. México: Universidad Iberoamericana, 2000. Impreso.

Grossberg, Lawrence. "Stuart Hall sobre raza y racismo: estudios culturales y la práctica del contextualismo". Tabula Rasa 5 (2006): 46-65. Impreso.

Lipovetsky, Gilles y Jean Serroy. L'esthétisation du monde. Vivre à l'âge du capitalisme artiste. Paris: Gallimard, 2013. Impreso.

Maffesoli, Michel. En el crisol de las apariencias. México: Siglo xxi, 2007. Impreso.

Méndez, Constanza. "Comunicación e identidad: una aproximación al estudio del consumo". Universitas Humanística 64 (2007): 291-305. Web.

Miller, Daniel. "Individuals and the aesthetic of order". Anthropology and the individual. A material culture perspective. Ed. Daniel Miller. Oxford: Berg, 2009. 3-24. Impreso.

Morales Tejada, Aida. "El universo material de la vida doméstica de la élite de Santiago de Cuba entre 1830 y 1868 ”. Historia Crítica 38 (2009): 96-121. Impreso.

Ochs, Elinor y Lisa Capps. "Narrating the self”. Annual Review of Anthropology 25 (1996): 19-43. Impreso.

Sennett, Richard. El artesano. Barcelona: Anagrama, 2012. Impreso.

Recibido: 26 enero 2015

Aceptado: 29 marzo 2016 